\title{
Aspectos éticos relacionados con las remuneraciones en odontología
}

Leonor Palomer Roggerone ${ }^{1}$

Resumen: Existen diferentes modalidades para recibir las remuneraciones en carreras liberales como odontología. En general, el pago se hace por acciones realizadas o por el tiempo dedicado a ejecutarlas. En algunos casos el monto del pago está directamente relacionado con el diagnóstico realizado al paciente. ¿Existe un conflicto de interés en alguna de las modalidades? Para responder esta pregunta se realizó una búsqueda bibliográfica sobre el tema, encontrándose escasa literatura al respecto. A partir de lo revisado podemos concluir que la existencia del conflicto de interés no es el problema, la resolución del mismo es el que aporta la dimensión ética al tema de las remuneraciones.

Palabras clave: remuneraciones, odontología, salario, pago por servicio

\section{Ethical aspects related to dentistry remunerations}

Abstract: There are different modalities to receive remunerations in liberal careers such as dentistry. In general, payment is done for actions carried out by time dedicated to execute them. In some cases, the amount of payment is directly related to the diagnosis of the patient. There is a conflict of interest in some of these modalities? To answer this question a bibliographical search was carried out on the topic, finding scarce literature on it. From the revised material, we can conclude that the problem is not the existence of a conflict of interest, but what brings an ethical dimension to the topic of remunerations is its resolution.

Key Words: remunerations, dentistry, salary, fee by service

\section{Aspectos éticos relacionados com as remunerações em odontologia}

Resumo: Existem diferentes modalidades para receber as remuneraçôes em carreiras liberais como odontologia. Em geral, o pagamento se faz por açóes realizadas ou pelo tempo dedicado a executá-las. Em alguns casos o montante do pagamento está diretamente relacionado com o diagnóstico realizado ao paciente. Existe um conflito de interesse em alguma das modalidades? Para responder esta pergunta se realizou uma busca bibliográfica sobre o tema, encontrando-se escassa literatura a respeito. A partir do que foi revisado podemos concluir que a existência do conflito de interesse não é o problema, a resolução do mesmo é o que aporta a dimensão ética ao tema das remuneraçóes.

Palavras-chave: remuneraçóes, odontologia, salário, pagamento por serviço

\footnotetext{
${ }^{1}$ Cirujano Dentista. Profesor Asistente Adjunto, Carrera de Odontología, Facultad de Medicina Pontificia Universidad Católica de Chile, Chile

Correspondencia: 1palomer@uc.cl
} 


\section{Introducción}

Desde la antigüedad se entiende por "honorarios" los emolumentos o estipendios que en justa retribución perciben por sus servicios las personas que se dedican a la práctica de las profesiones llamadas "liberales", como es la odontología.

En Chile, hasta mediados de los años 80, los cirujanos dentistas habitualmente tenían dos modalidades de trabajo: podían ser contratados por alguna institución pública o privada y/o ejercían en su clínica privada. En el primer caso tenían un contrato de trabajo con un sueldo y se les pagaban las leyes sociales correspondientes. Si se dedicaban al ejercicio privado, ellos estipulaban directamente con sus pacientes los valores a cobrar por los tratamientos realizados.

Con el advenimiento de las Instituciones de Salud Previsional (ISAPRES) y la formación de centros dentales que ofrecen atención a grupos de pacientes, con descuentos y convenios especiales, se popularizó la modalidad de trabajo a honorarios, en la cual existe un mediador que cobra por las atenciones y tratamientos y el odontólogo recibe un porcentaje de lo cobrado, en un monto a convenir.

La Organización Internacional del trabajo (OIT, 1985) reconoce dos principales modalidades: Sistema de Remuneración por Resultado y Sistema de Remuneración por Tiempo.

En los diferentes países existen sistemas de remuneraciones particulares para el pago de los honorarios a los cirujanos dentistas. En general, lo más similar al pago de un porcentaje por acción realizada es lo denominado "fee for service", en que se remunera por cada unidad de trabajo ejecutado. El pago por el tiempo dedicado a la atención de pacientes se conoce como salario. También, en otros países es muy popular el sistema llamado "capitation", en que el pago es efectuado por cada paciente que es atendido por el profesional, independientemente de lo realizado.

En este contexto podemos preguntarnos: ¿tiene alguna implicancia ética la manera en que los profesionales reciben sus remuneraciones? ¿Se genera un conflicto de interés en el profesional, cuando su remuneración depende del diagnóstico que realice al paciente? Nuestro objetivo es investigar en la literatura si se ha descrito alguna relación entre el modo de recibir los honorarios y el comportamiento moral de los odontólogos.

\section{Método}

Se realizó una búsqueda en Internet, ingresando a través del sitio del Sistema de Bibliotecas de la Pontificia Universidad Católica de Chile (SIBUC) a un metabuscador que permite acceder a revistas electrónicas, bases de datos y un catálogo en línea, que contiene libros y revistas impresas. Se relacionaron los términos "salary", "fee for service", "dental" y "ethics", y se seleccionaron los artículos relevantes, que fueran atingentes al tema y utilizaran una metodología apropiada.

Debido a la similitud del sistema de pago y por no existir muchos estudios realizados en odontólogos, se incluyeron investigaciones realizadas en médicos y que tenían objetivos similares.

\section{Resultados}

Como resultado de la búsqueda se seleccionaros 11 trabajos sobre el tema, incluyendo investigaciones realizadas en médicos y dentistas. Resumimos las principales conclusiones de esos estudios:

En 2009, Gosden T, Forland F, Kristiansen I, y colaboradores(1) realizaron una revisión sistemática comparando los sistemas de capitation, salary, fee-for-service y los sistemas mixtos de pago en cuanto a su efecto en el comportamiento de los médicos de atención primaria, midiendo el grado de satisfacción con el ambiente de trabajo, el costo, cantidad, tipo y modelo de la atención, la equidad de la misma y el estado de salud y satisfacción del paciente. Revisaron cuatro estudios en los que se incluían 640 médicos de atención primaria y más de 6.400 pacientes. En su trabajo concluyen que hay cierta evidencia de que los médicos entregan una gran cantidad de servicio de atención primaria cuando trabajan bajo el sistema de fee for service, comparado con capitation y salary, aunque los efectos en el largo tiempo no están claros. Sin embargo, no hay evidencia respecto de otras variables, como el estado de salud de los pacientes o una comparación del impacto 
relativo del salario versus la capitation. Textualmente concluyen: "Existe cierta evidencia que sugiere que la forma de pago de los médicos de atención primaria afecta su comportamiento, pero no podemos generalizar la causa de los hallazgos".

En otro trabajo publicado en 1999 por T. Gosden, L. Pedersen y D. Torgerson(2) se analizaron estudios publicados y no publicados en los que se comparaba el comportamiento entre médicos generales asalariados y los que recibían otros tipos de pago, y que explicitaban resultados en cuanto a la conducta de los profesionales. En su revisión seleccionaron veintitrés publicaciones y concluyen que son escasos los estudios de calidad que comparen el sistema de pago de los profesionales con la eficiencia o equidad de sus tratamientos. Sus resultados mostraron que el pago de salarios reduce la actividad de los médicos generales, comparados con los que reciben pago por servicio, y que el sistema de capitación tiene un efecto similar, aunque menor. También los resultados de la revisión sugieren que, si el ahorro de recursos es un efecto buscado, el pago de honorarios es más efectivo en lograr esto que el pago por servicio y la capitación.

Según se menciona en el trabajo publicado por Johan Hartshorne y Thomas K. Hasegawa(3), desde que los odontólogos están en una posición de tener ganancias financieras por sus recomendaciones profesionales, ellos están en riesgo de tener conflictos de interés: con un sobrediagnóstico reciben mayores ingresos. Esto sería favorecido por la baja de las tarifas odontológicas, la demora en los pagos, exceso de dentistas y el aumento del precio de los materiales y equipamiento dental. Además, durante mucho tiempo no han existido regulaciones que aseguren lo apropiado o necesario de un tratamiento dental. La sociedad confía en que sus profesionales pondrán el beneficio de aquellos que tratan por sobre sus propios intereses.

Según se describe en el trabajo de F. J. Sámano y G. Fernández(4), al aparecer los intermediarios en el pago de honorarios entre el profesional y su paciente se limitó la libertad de decisión de ambos, emergiendo rápidamente como elementos centrales para la toma de decisiones médicas los criterios de eficiencia social e institucional, así como la preocupación por los costos en la atención médica. Esta situación llevaría a que la imagen de los médicos se haya deteriorado y devaluado, ya que están a merced de "las fuerzas del mercado", "de la oferta y la demanda”, del pragmatismo lucrativo de los intermediarios y los terceros pagadores, incluyendo al Estado.

Stefan Greß, Diana M. J. Delnoij y Peter P. Groenewegen(5) publicaron un trabajo en el que concluyen que los sistemas de pago e incentivos financieros influyen en el comportamiento de los proveedores de atención primaria. Sin embargo, cómo exactamente y en qué medida, depende de una serie de otras influencias, tales como las limitaciones éticas y profesionales y el contexto del sistema de salud. Los sistemas de pago no son el factor más influyente que dirige el comportamiento profesional, pero es el más fácil de modificar por los responsables políticos.

Martin Chalkleya y Colin Tilleyb(6) estudiaron el comportamiento de los dentistas en el Servicio Nacional de Salud Británico (NHS); estos profesionales trabajan bajo una variedad de sistemas de remuneración, lo que implica diferencias en el monto que efectivamente reciben por cada prestación realizada. Compararon a los dentistas independientes con los asalariados, y su estudio muestra diferencias en el tratamiento ofrecido al paciente, dependiendo del tipo de remuneración recibida por el profesional. Sus resultados apoyan el hecho de que los incentivos financieros son determinantes importantes del comportamiento médico.

Mary Ann Baily(7), en su publicación de 2004, afirma que el sistema de salud debe de alguna manera guiar a los médicos a practicar una medicina consciente de los costos, en el sentido de que los pacientes obtengan ni mucho ni demasiado poco ni en cantidad o calidad de la atención.

Para Mariangela Leal Cherchiglia(8) el pago por tiempo es el "ideal", pero deben adoptarse mecanismos que incentiven la producción, mejoren la calidad de los servicios y, finalmente, el propio valor real de los sueldos.

En Noruega, J. Grytten y R. Sørensen(9) tienen una opinión diferente. En su estudio concluyeron 
que no existiría inducción de demanda de servicios por parte del médico de atención primaria en Noruega y que las normas profesionales desempeñan un papel importante en el control de la buena conducta de un médico.

Alan R. Nelson(10) expone, por otra parte, que los encargados de los pagos a los profesionales tienen una responsabilidad moral y ética al implementar programas en los que se premia la eficiencia, definida por la relación entre el costo de la atención y el rendimiento, promoviendo recompensas económicas o incentivando a los pacientes a escoger a los profesionales que serían más eficaces, al utilizar menos recursos.

Mark A. Hall y colaboradores(11) realizaron un estudio para medir si la entrega de información a los pacientes, acerca de cómo se calculaba la remuneración de sus médicos en los sistemas de pago por servicio y capitación, afectaba su confianza en ellos, y concluyeron que saber cómo son remunerados los profesionales no afecta la credibilidad ante sus pacientes. Cabe destacar que los bonos que gratificaban a los profesionales eran entregados como premio por el ahorro de costos, satisfacción del paciente e implementación de medidas de prevención.

\section{Discusión}

No existe mucho material sobre el tema de las implicancias éticas de las remuneraciones en el área de la odontología, en cuanto a si tienen una influencia en los valores morales de los profesionales y a su actuar frente a los pacientes. Podemos extrapolar información desde trabajos realizados respecto de médicos en situaciones similares.

Situar al profesional en un conflicto de interés, al depender su pago del número de acciones que realice en su paciente, condiciona una situación que pone a prueba el comportamiento moral del odontólogo. La existencia del conflicto de interés no es el problema, la resolución del mismo es el que aporta la dimensión ética al tema de las remuneraciones.
Existen riesgos de una mala resolución del conflicto de interés en los diferentes tipos de remuneración, ya sea por un sobrediagnóstico, discriminación de pacientes, selección de acciones a realizar u otro; en general, cuando no se cumpla con poner el bienestar del paciente como centro del quehacer profesional.

La formación moral de los profesionales es la única herramienta que puede asegurar un comportamiento ético adecuado y un resguardo de los pacientes, además de garantizar el prestigio y credibilidad de una profesión como la odontología.

Como conclusiones de esta investigación podemos destacar que:

Todas las formas de pago de remuneraciones pueden provocar conflictos éticos.

- El pago de honorarios, o fee for service, puede incentivar el aumento del número de servicios realizados y proporcionar más prestaciones de las necesarias a los pacientes.

- Por otro lado, el sistema de capitación puede incentivar la selección de pacientes de riesgo bajo y el subtratamiento.

- El pago de salario puede estimular la minimización de costos personales, como el esfuerzo, produciéndose un subtratamiento.

Por lo tanto, no existe un sistema ideal. El reto es alcanzar formas de pago flexibles, efectivas, con profesionales comprometidos y que proporcionen atención de calidad.

Para una buena resolución de los conflictos de interés que puedan generarse por la forma de recibir las remuneraciones, la formación valórica del odontólogo es primordial.

Al terminar, podemos citar el texto del American College of Dentists: "Dental Managed Care in the Context of Ethics" (12), que propugna que los intereses económicos propios de los dentistas no deben ser puestos por encima de los objetivos a los que se aspira: la compasión, la justicia y la beneficencia. 


\section{Referencias}

1. Gosden T, Forland F, Kristiansen I, Sutton M, Leese B, Giuffrida A, et al. Capitation, salary, feefor-service and mixed systems of payment: effects on the behaviour of primary care physicians. Cochrane Database of Systematic Reviews 2000, Issue 3. Art. No CD002215. DOI: 10.1002/14651858. CD002215.

2. Gosden T, Pedersen L, Torgersen D. How should we pay doctors? A systematic review of salary payments and their effect on doctor behaviour. Quarterly Journal of Medicine 1999; 92(1): 47-55.

3. Hartshorne J, Hasegawa TK. Overservicing in dental practice-ethical perspectives. J S Afr Dent Assoc October 2003; 58(9): 364-369.

4. Sámano FJ, Fernández G. Los honorarios médicos en la era de los terceros pagadores. An Med Asoc Med Hosp ABC 2002; 47(2): 118-127

5. Greß S, Delnoij D, Groenewegen P. Managing primary care behavior through payment systems. Primary care in the driver's seat. European Observatory on Health Systems. Available from URL: http://www.euro.who.int/Document/E87932_11.pdf

6. Chalkley M, Tilley C. Treatment Intensity and Provider Remuneration: dentists in the British National Health. Service Health Econ 2006; 15: 933-946.

7. Baily MA. Ethics, Economics, and Physician Reimhursement. The Mount Sinai Journal of Medicine, September 2004; 71(4): 231-235.

8. Cherchiglia ML. Sistemas de Pago y Práctica Médica: teoría y evidencias empíricas. Programa de Desarrollo de Recursos Humanos, División de Desarrollo de Sistemas y Servicios de Salud. Organización Panamericana de la Salud/Organización Mundial de la Salud, 2002. Available from URL: http://www.esmed.com.ar/download/postgrado/gestion_auditoria/bibliografia/modulo_10/sistpago_practmed.pdf

9. Grytten J, Sørensen R. Type of contract and supplier-induced demand for primary physicians in Norway. Journal of Health Economics 2001; 20: 379-393.

10. Nelson AR. Pay-for-performance programs: Ethical questions and unintended consequences. $\mathrm{Cu}$ rrent Clinical Practice October 2007; 1(1): 16-18.

11. Mark A. et al. How Disclosing HMO Physician Incentives Affects Trust. Health Affairs 2002; 21(2): 197-206

12. American College of Dentists: Dental Managed Care in the Context of Ethics. Journal of the American College of Dentists Winter 1996; 63(4): 19-21.

Recibido: 22 de marzo de 2011

Aceptado: 25 de junio de 2011 